\title{
The contribution of susceptibility-weighted imaging (SWI) in the central nervous system superficial siderosis' evaluation in 3 Tesla magnetic resonance imaging (MRI)
}

\author{
Bünyamin Güney, İbrahim Önder Yeniçeri, Emrah Doğan, Neşat Çullu \\ Muğla Sıtkı Koçman University, Faculty of Medicine, Deparment of Radiology \\ Department of Radiology, Muğla Sıtkı Koçman University Medical Faculty, Muğla, Turkey
}

\begin{abstract}
Objective. The aim of this study is to evaluate the magnetic resonance imaging (MRI) findings of central neural system (CNS) superficial siderosis cases and the diagnostic contribution of the susceptibility-weighted imaging (SWI) sequence to conventional imaging.

Method. TSE T2-weighted and SWI-MRI of 26 patients diagnosed as CNS-superficial siderosis (CNS-SS) were retrospectively evaluated with 3-Tesla MRI. The localization and type of involvement of SS were reviewed.

Results. The CNS-SS were divided into two categories as central amyloid angiopathy-SS (CAA-SS) and non-central amyloid angiopathy-SS (non-CAA-SS). In non-CAA cases, the involvement was typical (classic) in 5 cases and atypical in 9 cases. In 12 of these cases (85.7\%), SS findings were observed on both turbo spine echo (TSE) T2 images and SWI imaging, while in 2 cases (14.3\%) SS was detected only on SWI images. In 7 of the CAA-SS cases, involvement was focal type SS (58.33\%), while 5 cases had diffuse type SS (41.67\%) involvement. In the vast majority of cases $(n=10)$ of this type of SS, involvement was detected only in SWI images, while siderosis was not detected in TSE T2 images. In addition, occult cerebral vascular malformation accompanying SS, which can be observed only in the SWI sequence, was found in a total of 4 cases. In the cross-matching statistical analysis performed between CAA-SS and non-CAA-SS groups and subgroups, SWI was found to be significantly superior to T2 in detecting SS in the CAA-SS group (p:0,007).

Conclusions. SWI imaging was superior in detecting SS and detecting cerebral occult vascular malformation in CAA-SS cases. Although the detectability of SS by SWI was high in other groups, no statistically significant difference was found. Under these circumstances, we think that it will be beneficial to add SWI imaging to the routine imaging protocol in cases with suspected CNS-SS.
\end{abstract}

Keywords: superficial siderosis, amyloid angiopathy, SWI, 3-Tesla MRI

\section{INTRODUCTION}

The central neural system-superficial siderosis (CNS-SS) is a rare pathological process characterized by the accumulation of hemosiderin in the subarachnoid space, leptomeninges and the superficial layers of the cerebral, cerebellar parenchyma or spinal cord due to various etiological reasons. The main cause of superficial siderosis (SS) is recurrent subarachnoid hemorrhage. Nowadays, it is diagnosed with increasing frequency due to advances in magnetic resonance imaging (MRI) (T2* and SWI imaging). There are many classifications in the literature according to etiological factors, clinical and localization, and involvement pattern. SS can be classified as cerebral amyloid angiopathy superficial siderosis (CAA-SS) and non-CAA-SS according to etiological reason and typical and atypical according to the clinical findings and the location. Additionally, during the recent years, new cortical type SS definition, which is divided into two categories as focal and diffuse types, mostly associated with CAA and/or Alzheimer's disease, has been also used 
(1). Although there are some studies and case reports about SS in the literature, studies that included classifications of SS are limited $(2,3)$. The aim of this study is to evaluate the MRI findings of central neural system (CNS) superficial siderosis cases and the diagnostic contribution of the susceptibility-weighted imaging (SWI) sequence to conventional imaging.

\section{MATERIAL AND METHODS}

\section{Study population}

Ethical approval with 200148/2020 number was obtained from Muğla Sıtkı Koçman University's human research ethics committee. In G power analysis, for $80 \%$ power and with critical t power: 1.7056179 , sample size was calculated as 26 . We retrospectively reviewed 49 patients diagnosed as superficial siderosis in the last 4 years. 23 patients without the SWI sequence were excluded from the study and 26 patients (mean age $59 \pm 18$ years 9 , range, 1-88 years,14 Male and 12 Female) were included in the study. Our patients were divided into two groups as non-CAA SS and CAA-SS according to modified Boston criteria considering clinical findings, age, and, and the anatomical involvement location. 12 patients (mean age 71,7 $\pm 8,16$ years, range, 59-88 years, 5 males and 7 females) were in the CCA-SS group, while 14 patients were in the non-CCA-SS group. 12 of patients were included in CCA-SS group (mean age 71,7 $\pm 8,16$ years, range, 5988 years, 5 males and 7 females), while 14 of patients group (mean age 48,1 \pm 18 ,9 years, range, 1-81 years, 9 males and 5 females) were in non-CCA-SS group.

\section{MRI protocols}

3 Tesla MR device (Siemens Skyra 3Tesla, 2016, Germany) were used for MRI. Applied technical parameters were as follow: T2 TSE: TR/TE: 3540/101 ms, matrix: 312x384 mm, NEX: 2 slice thickness: 5 mm. for SWI sequence: TR/TE: $27 / 20 \mathrm{~ms}$, matrix: 232x256 mm, NEX: 1, slice thickness $3 \mathrm{~mm}$.

\section{Data analysis}

The double-blinding method was used. Two experienced radiologists who have 14 and 12 years of experience in neuroradiology retrospectively evaluated the MRI on the PACS workstation. In case of contradictory results, the images were evaluated by four radiologists together. Non-CAA SS cases were grouped as classical typical involvement (predilection to the infratentorial region and spinal cord involvement) and atypical (supratentorial region focal or diffuse involvement). In the atypical group, the lesion limited to an area or lobe was considered as focal type. If SS is affected other lobes or both cerebral hemispheres, it was accepted as diffuse type. According to Boston criteria, Focal type SS was defined as a lesion limited to three cerebral sulci, while the diffuse type was defined as four sulci and more widespread involvement.

For both patient groups (CAA SS and Non-CAA SS); it was evaluated as negative (-) if the lesion could not be detected in the MRI sequences, detectable (+), appearance sharp and wide (++). Then, SWI sequences were evaluated and their contribution to the detectability of lesions was separately evaluated.

\section{Statistical analysis}

The data were enrolled on Microsoft office excel file (Excel 2019, Microsoft). Statistical software (SPSS, version 22.0, IBM) was used for analysis. Continuous variables were expressed as mean ( \pm SD) values. The classic-atypical subgroups and focal-diffuse types based on the Non-CAA-SS and CAA-SS main groups were compared according to the TSE and SWI sequences susceptibility. Pearson's chisquared test $\left(\chi^{2}\right)$ or Fisher exact test were used for comparison. $\mathrm{P}<0.05$ values were considered statistically significant.

\section{RESULTS}

12 of 26 patients were diagnosed as CAA-SS according to the last classification defined in the literature (Modified Boston Criteria). The focal type was found in 7 patients (58.33\%), diffuse type in 5 patients $(41.67 \%)$ in the CCA-SS group. SWI was successful in diagnosis in all patients while T2 SE images were not effective in 10 cases (Table 1 ).

In non-CAA cases, involvement was typical (classic) in 5 cases and atypical in 9 cases. Both TSE T2 images and SWI imaging showed SS in 12 of these cases ( $85.7 \%$ ), while 2 cases (14.3\%) were detectable only with SWI images. In classical (typical) type SS cases, involvement was observed predominantly in the posterior fossa, as well as in the spinal cord and cerebral hemispheres. As clinical findings, all had slowly progressed sensorineural hearing impairment, cerebellar ataxia, and corticospinal system disorders. Other patients had focal neurological symptoms (Table 2).

In almost the whole of the cases, SS findings were more pronounced and widespread on SWI images compared to TSE T2 images. In addition, occult cerebral vascular malformation accompanying SS, which can be seen only in the SWI sequence and sometimes associated with SS alone in the literature, was detected in 4 cases. There was a statistically significant difference in favor of SWI positivity in the CAA-SS group when TSE and SWI were com- 
TABLE 1. CAA-associated superficial siderosis

\begin{tabular}{|c|c|c|c|c|c|c|c|c|c|}
\hline \multirow[t]{2}{*}{ Number } & \multirow[t]{2}{*}{ Age } & \multirow[t]{2}{*}{ Gender } & \multirow[t]{2}{*}{ SS type } & \multirow[t]{2}{*}{ SS localization } & \multirow{2}{*}{$\begin{array}{l}\text { Clinical } \\
\text { findings }\end{array}$} & \multicolumn{2}{|c|}{ SS detectability } & \multicolumn{2}{|c|}{$\begin{array}{c}\text { Additional vascular } \\
\text { anomaly }\end{array}$} \\
\hline & & & & & & FSE T2 & SWI & FSE T2 & SWI \\
\hline 1 & 71 & $\mathrm{M}$ & Diffuse & Bilateral fronto-parietal & PNF & Negative & Positive & No & No \\
\hline 2 & 59 & $\mathrm{M}$ & Diffuse & Right parieto-occipital & PNF & Negative & Positive & No & No \\
\hline 3 & 81 & $\mathrm{~F}$ & Diffuse & Left fronto-temporal & PNF & Negative & Positive & No & $\mathrm{DVA}+\mathrm{CCM}$ \\
\hline 4 & 67 & $\mathrm{~F}$ & Diffuse & Bilateral fonto-parietal & PNF & Negative & Positive & No & No \\
\hline 5 & 74 & $\mathrm{M}$ & Diffuse & Right fronto-parietal & PNF & Negative & Positive & No & No \\
\hline 6 & 67 & $\mathrm{~F}$ & Focal & Right parietal & FNF & Negative & Positive & No & No \\
\hline 7 & 71 & M & Focal & Left parietal & FNF & Negative & Positive & No & No \\
\hline 8 & 88 & $\mathrm{M}$ & Focal & Right perirolandic region & FNF & Positive & 2xPositive & No & No \\
\hline 9 & 79 & $\mathrm{~F}$ & Focal & Left occipital & FNF & Negative & Positive & No & No \\
\hline 10 & 72 & $\mathrm{~F}$ & Focal & Right parietal & FNF & Positive & 2xPositive & No & DVA \\
\hline 11 & 64 & $\mathrm{M}$ & Focal & Left parietal & FNF & Negative & Positive & No & No \\
\hline 12 & 67 & $M$ & Focal & Left temporal & FNF & Negative & Positive & No & No \\
\hline
\end{tabular}

SS - superficial siderosis, CAA - cerebral amyloid angiopathy, PNF-progressive neurological findings, FNF-focal neurological findings, FSE T2W - Fast Spin Echo T2 Weighted, SWI - susceptibility weighted imaging, CCM - cerebral cavernous malformation, DVA - developmental venous anomaly

TABLE 2. Non-CAA superficial siderosis

\begin{tabular}{|c|c|c|c|c|c|c|c|c|c|c|}
\hline \multirow[b]{2}{*}{ Number } & \multirow[b]{2}{*}{ Age } & \multirow[b]{2}{*}{ Gender } & \multirow[b]{2}{*}{ SS type } & \multirow[b]{2}{*}{ SS localization } & \multirow[b]{2}{*}{ Etiology } & \multirow{2}{*}{$\begin{array}{l}\text { Clinical } \\
\text { Findings }\end{array}$} & \multirow{2}{*}{\begin{tabular}{|c|} 
SS \\
Detectability \\
FSE T2W \\
\end{tabular}} & \multicolumn{3}{|c|}{ Additional Vascular Anomaly } \\
\hline & & & & & & & & SWI & $\begin{array}{c}\text { FSE } \\
\text { T2W }\end{array}$ & sWI \\
\hline 1 & 38 & M & $\begin{array}{c}\text { Classical (typical) } \\
\text { involvement }\end{array}$ & $\begin{array}{c}\mathrm{CH}, \mathrm{Crb}, \mathrm{BS}, \\
\text { IAC, SC. }\end{array}$ & posttraumatic & $\begin{array}{l}\text { Classical } \\
\text { clinical } \\
\text { findings }\end{array}$ & Positive & 2xPositive & No & No \\
\hline 2 & 62 & $\mathrm{~F}$ & $\begin{array}{c}\text { Classical (typical) } \\
\text { involvement }\end{array}$ & $\begin{array}{c}\mathrm{CH}, \mathrm{Crb}, \mathrm{BS} \\
\text { IAC, SC. }\end{array}$ & idiopathic & $\begin{array}{l}\text { Classical } \\
\text { clinical } \\
\text { findings }\end{array}$ & Positive & 2xPositive & No & No \\
\hline 3 & 46 & $\mathrm{~F}$ & $\begin{array}{c}\text { Classical (typical) } \\
\text { involvement }\end{array}$ & $\begin{array}{c}\mathrm{CH}, \mathrm{Crb}, \mathrm{BS}, \\
\text { IAC, SC. }\end{array}$ & $\begin{array}{l}\text { operated hemorrhagic } \\
\text { pituitary adenoma }\end{array}$ & $\begin{array}{l}\text { Classical } \\
\text { clinical } \\
\text { findings }\end{array}$ & Positive & 2xPositive & No & $\begin{array}{l}\text { Multipl } \\
\text { CCM }\end{array}$ \\
\hline 4 & 54 & M & $\begin{array}{c}\text { Classical (typical) } \\
\text { involvement }\end{array}$ & $\mathrm{Crb}, \mathrm{BS}, \mathrm{IAC}$ & $\begin{array}{l}\text { RCC, hemorrhagic } \\
\text { brain metastasis }\end{array}$ & $\begin{array}{l}\text { Classical } \\
\text { clinical } \\
\text { findings }\end{array}$ & Positive & 2xPositive & No & No \\
\hline 5 & 67 & M & $\begin{array}{c}\text { Classical (typical) } \\
\text { involvement }\end{array}$ & $\mathrm{Crb}, \mathrm{BS}, \mathrm{IAC}$ & $\begin{array}{l}\text { Neurocysticercosis } \\
\text { rosemosis stage }\end{array}$ & $\begin{array}{l}\text { Classical } \\
\text { clinical } \\
\text { findings }\end{array}$ & Positive & 2xPositive & No & No \\
\hline 6 & 45 & $\mathrm{~F}$ & $\begin{array}{c}\text { Atypical } \\
\text { involvement- } \\
\text { diffuse }\end{array}$ & $\begin{array}{c}\text { Bilateral } \\
\text { fronto-parietal }\end{array}$ & $\begin{array}{l}\text { Bilateral trolard vein } \\
\text { thrombosis }\end{array}$ & PNF & Positive & 2xPositive & No & No \\
\hline 7 & 31 & M & $\begin{array}{c}\text { Atypical } \\
\text { involvement- } \\
\text { diffuse }\end{array}$ & $\begin{array}{l}\text { Bilateral } \\
\text { parieto- } \\
\text { occipital }\end{array}$ & posttraumatic & PNF & Positive & 2xPositive & No & No \\
\hline 8 & 56 & M & $\begin{array}{c}\text { Atypical } \\
\text { involvement-focal }\end{array}$ & Right frontal & operated glial tumor & FNF & Negative & Positive & No & $\begin{array}{l}\text { Right } \\
\text { Frontal } \\
\text { DVA }\end{array}$ \\
\hline 9 & 62 & $\mathrm{~F}$ & $\begin{array}{c}\text { Atypical } \\
\text { involvement-focal }\end{array}$ & Right parietal & $\begin{array}{l}\text { chronic hemorrhagic } \\
\text { infarction }\end{array}$ & FNF & Positive & 2xPositive & No & No \\
\hline 10 & $\begin{array}{c}6 \\
\text { month }\end{array}$ & $\mathrm{F}$ & $\begin{array}{c}\text { Atypical } \\
\text { involvement-focal }\end{array}$ & Subependymal & $\begin{array}{c}\text { sequela of } \\
\text { intraventricular } \\
\text { bleeding }\end{array}$ & FNF & Negative & Positive & No & No \\
\hline 11 & 81 & $M$ & $\begin{array}{c}\text { Atypical } \\
\text { involvement-focal }\end{array}$ & Right Frontal & $\begin{array}{c}\text { Operated Intracranial } \\
\text { Aneurysm }\end{array}$ & FNF & Positive & 2xPositive & No & No \\
\hline 12 & 49 & M & $\begin{array}{c}\text { Atypical } \\
\text { involvement-focal }\end{array}$ & Left parietal & $\begin{array}{l}\text { Cerebral cavernous } \\
\text { malformation }\end{array}$ & FNF & Positive & 2xPositive & No & No \\
\hline 13 & 43 & $M$ & $\begin{array}{c}\text { Atypical } \\
\text { involvement-focal }\end{array}$ & Right frontal & $\begin{array}{l}\text { Cerebral cavernous } \\
\text { malformation }\end{array}$ & FNF & Positive & 2xPositive & No & No \\
\hline 14 & 39 & $\mathrm{M}$ & $\begin{array}{c}\text { Atypical } \\
\text { involvement-focal }\end{array}$ & $\begin{array}{l}\text { Right medial } \\
\text { temporal }\end{array}$ & posttraumatic & FNF & Positive & 2xPositive & No & No \\
\hline
\end{tabular}

SS - superficial siderosis, CAA - cerebral amyloid angiopathy, $\mathrm{CH}$ - cerebral hemispheres, Crb - cerebellum, iAC - internal acoustic channels, BS - brainstem, SC - spinal cord, PNF - progressive neurological findings, FNF - focal neurological findings, RCC - renal cell carcinoma, FSE T2W - Fast Spin Echo T2 Weighted, SWI - susceptibility weighted imaging, CCM - cerebral cavernous malformation, DVA - developmental venous anomaly 
pared [P: $0.007(\mathrm{p}<0.05)]$. In the CAA-SS group, the SWI sequence was interpreted as significantly effective in showing lesions compared to T2. In comparison to other groups, $\mathrm{p}$ was $>0.05$. There was no statistical difference in diagnosis between $\mathrm{T} 2$ and SWI in diffuse-focal and classical-atypical forms. In addition, there was no significant difference between T2 and SWI in the non-CAA group.

\section{DISCUSSION}

Susceptibility weighted imaging (SWI), also known as BOLD venographic imaging, is an MRI sequence that is quite sensitive to venous blood, hemorrhage and iron storage including paramagnetic, diamagnetic, and ferromagnetic materials. SWI uses a flow compensated, long echo, gradient recalled echo (GRE) pulse sequence to obtained images (1-4). Generally, 3 Tesla MR's sensitivity effects and detectability are better than 1.5 Tesla MRI devices (5).

The 'classical' or typical SS of the CNS affects the infratentorial region, acoustic nerves and spinal cord. It typically presents with slow progressed sensorineural hearing impairment, cerebellar ataxia, and corticospinal system findings. Although this type of SS is assumed to be caused by chronic intermittent or continuous minor bleeding into the subarachnoid space, a definite source of bleeding (CNS tumors, arteriovenous malformations, aneurysms, vasculitis, intradural surgery and trauma) has been revealed in only $50-75 \%$ of cases $(6,7)$. Non-CAA SS does not always present with this typical clinical finding or type of involvement. This group is defined as atypical SS. It can be limited with some areas in the cerebral or cerebellar region (localized or focal type), or involvement can be diffuse (diffuse SS) (8). Wang et al. reported that the SWI sequence can reveal earlier the presence of hemosiderin in the CSS-SS compared to the TSE T2 sequence, and can provide a more detailed and complete evaluation of SS (9). In our study, we found that the SWI sequence shows affected areas sharper and detailed in non-CAA SS (Figure 1, 2).

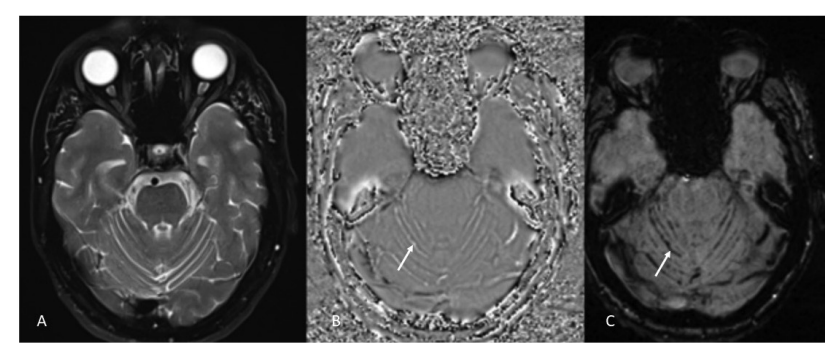

FIGURE 1. Axial T2 FSE (A), phase (B) and SWI images (C). The classical type superficial siderosis in both cerebellar hemispheres is visible only in SWI and phase images (white arrow).

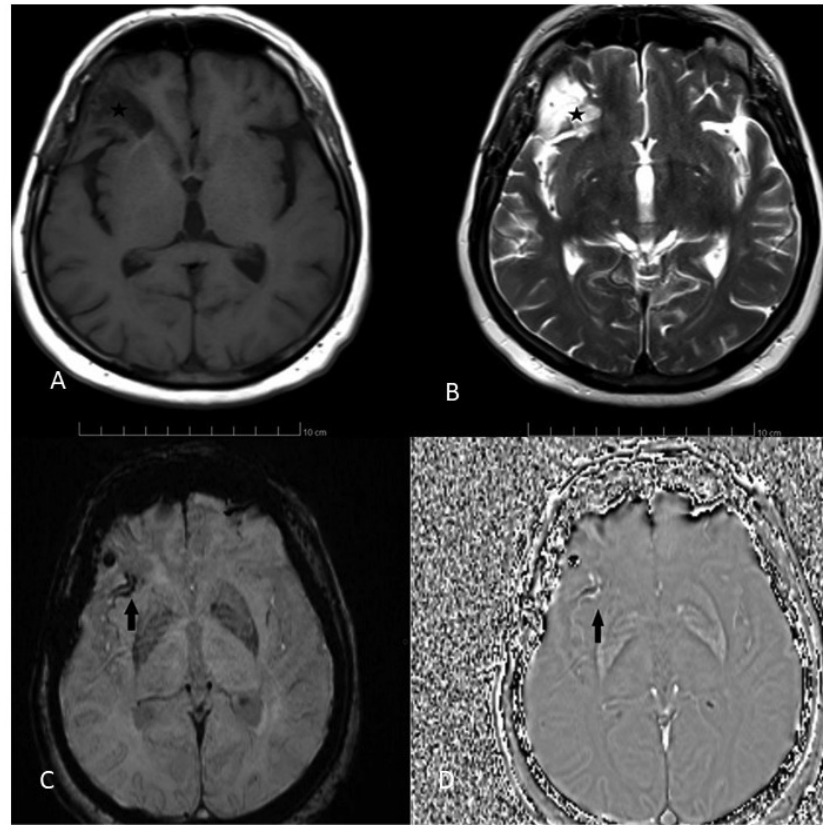

FIGURE 2. Postoperative bone and soft tissue changes in the right frontal region and sequela parenchyma loss and gliosis area (black star) in the frontal lobe on axial T1 FSE (A) and T2 FSE (B) images. Focal superficial siderosis findings (black arrow) at the posterior of the gliosis area in the SWI (C) and phase (D) images of the case. Superficial siderosis is seen unclear on the FSE T2 axial image, while the SWI is seen clearly and sharply on the image.

The second type of SS has been defined in the literature in the last 10-15 years, limited to the convexities of the supratentorial compartment and the cerebral hemispheres, and is called "cortical" superficial siderosis (CSS) (10). CSS has different potential causes and clinical findings compared to classical (typical) SS. This type of SS is more commonly referred to as CAA-associated SS (CAA-SS) and occurs in elderly patients as one of the keys finding of cerebral amyloid angiopathy, an age-related cerebral small vessel disorder (11-13). CAA-SS is associated with characteristic clinical symptoms, including transient focal neurological attacks. The SWI sequence is a very important and necessary MR sequence in the detection of cerebral microhemorrhages, which are diagnostic criteria for CAA according to the modified Boston criteria. In addition, the presence of cortical SS in the patients with CAA are an important sign for the risk of future intracerebral hemorrhage (14). In our study, while SS was not detectable on TSE T2 images in almost whole CAA-SS cases, it could be revealed on SWI images (Figure 3). For this group, the SWI sequence is essential for diagnosis, and the SS that can only be detected in this sequence is a significant risk factor for future bleeding. Therefore, the addition of SWI to routine imaging is vital for the detection of SS in SAA and Alzheimer's patients. Early diagnosis of SS is very important in discontinuation of anticoagu- 


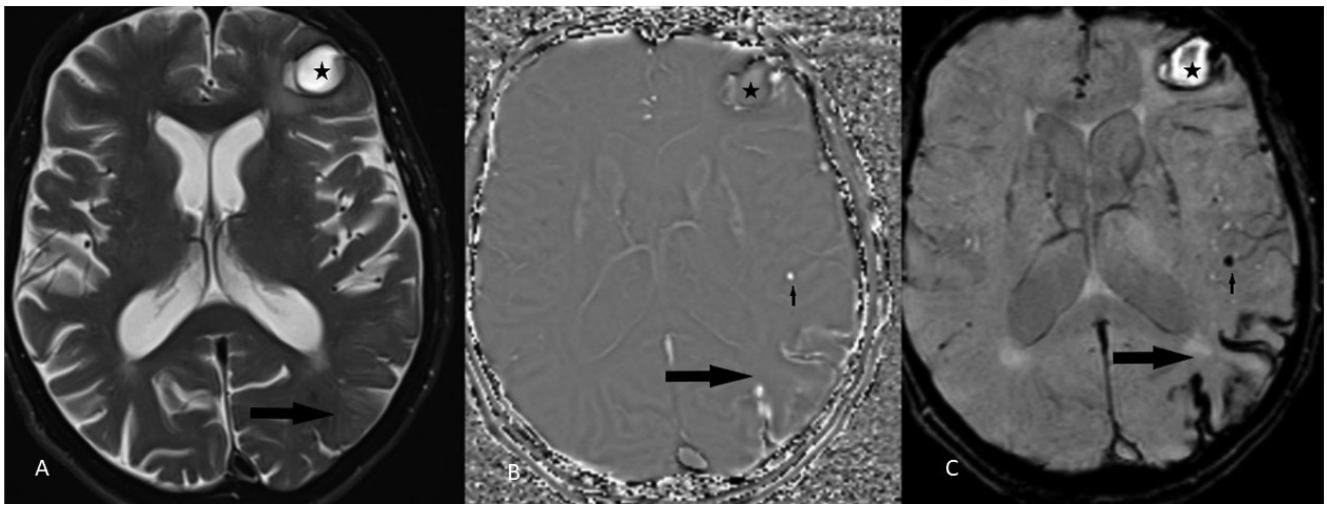

FIGURE 3. Display axial T2 FSE (A), phase (B) and SWI (C). Case with cerebral amyloid angiopathy, left frontal hematoma (star) and focal microhemorrhagic focus (small arrow) in parenchyma. Diffuse type cortical superficial siderosis (large arrow) involving 4 sulcus and more in the left temporooccipital region and microhemorrhagic parenchymal focus can only be seen in SWI and phase imaging, while the FSE T2 image cannot be seen.

lant therapy and initiation of iron chelate therapy such as deferiprone to prevent progression of siderosis symptoms. CAA SS can be detected at an early stage almost exclusively with the SWI sequence $(15,16)$.

Cerebral occult vascular malformations (lowflow vascular malformations like cavernous malformation (17), developmental venous anomaly and capillary telangiectasia) may be CNS-SS etiological causes (18). Li et al. reported three cases of SS associated with cerebral vascular malformations and attributed the cause of pathological hemosiderin accumulation to these lesions located near the subarachnoid spaces (19). In addition, in the literature, Ogura et al. reported an SS case due to cerebral cavernous malformation localized in the pineal region (20). In our study, we found occult cerebral vascular malformations that can only be seen on SWI images and associated with SS etiology in 4 cases (Figure 4 ).

The number of included studies was limited in terms of sample size, data availability, and methodologic quality. SWI sequence was not present in half of the diagnosed cases. For this reason, a large group was excluded from the study. However, since SS is a rare entity, the number of cases was in the acceptable range. The sufficiency of sample size was confirmed with the G power test. Nevertheless, it would be useful to expand current research with more detailed data and a larger study group in the future.

\section{CONCLUSIONS}

In CAA-SS, the SWI sequence was found to be statistically superior to T2 imaging in lesion detectability. Cerebral occult vascular malformations were able to be detected merely with SWI sequence. There was no statistically significant difference between T2 and SWI in non-CCA SS and its subgroups. However, the lesion detection rate of SWI was higher or equal to T2 in all groups. Under these circumstances, It is thought that it will be beneficial to add SWI imaging to the routine imaging protocol in cases with suspected CNS-SS.

Conflict of interest: none declared Financial support: none declared

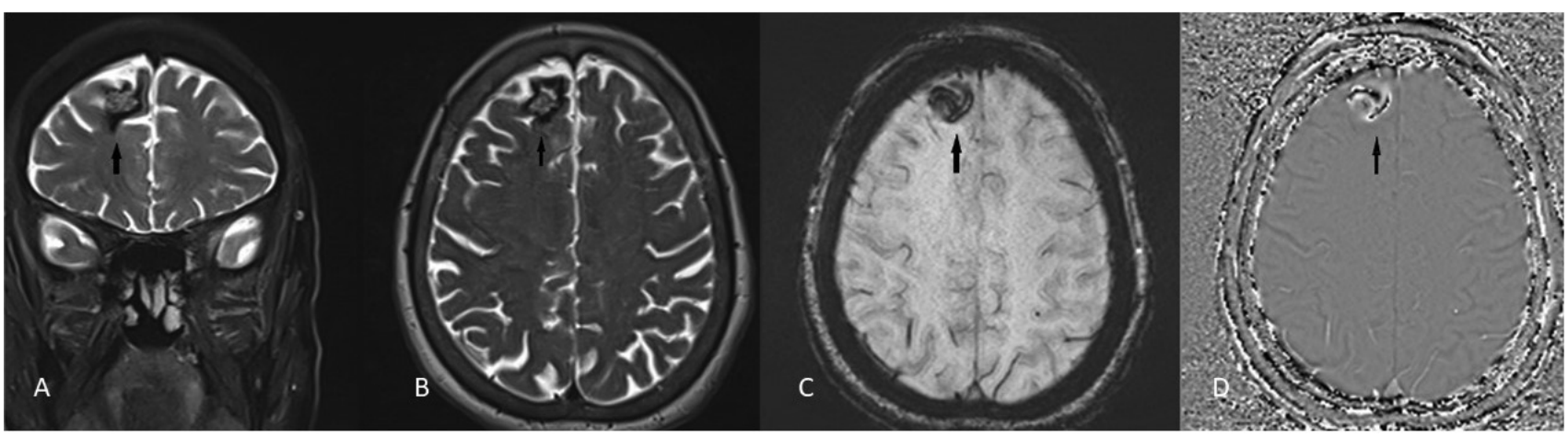

FIGURE 4. In the coronal T2 FSE (A) and axial T2 FSE (B) image, cavernous angioma in the right frontal region and focal superficial siderosis developed adjacent to it. Superficial siderosis is observed more sharply and clearly in SWI (C) and phase images (D) of the same case (arrows). 


\section{REFERENCES}

1. Charidimou A, Linn J, Vernooij MW, Opherk C, Akoudad S, Baron JC, Greenberg SM, Jäger HR, Werring DJ. Cortical superficial siderosis: detection and clinical significance in cerebral amyloid angiopathy and related conditions. Brain. 2015 Aug;138(Pt 8):2126-39.

2. Sehgal V, Delproposto Z, Haacke EM, Tong KA, Wycliffe N, Kido DK, et al. Clinical applications of neuroimaging with susceptibility-weighted imaging. J Magn Reson Imaging. 2005;22:439-50.

3. Haacke EM, DelProposto ZS, Chaturvedi S, Sehgal V, Tenzer M, Neelavalli J, et al. Imaging cerebral amyloid angiopathy with susceptibility-weighted imaging. AJNR Am J Neuroradiol. 2007; 28:316-7.

4. de Souza JM, Domingues RC, Cruz LC, Domingues FS, lasbeck T, Gasparetto EL. Susceptibility-weighted imaging for the evaluation of patients with familial cerebral cavernous malformations: a comparison with t2-weighted fast spin-echo and gradient-echo sequences. AJNR Am J Neuroradiol. 2008;29:154-8.

5. Stehling $\mathrm{C}$, Wersching $\mathrm{H}$, Kloska SP, Kirchhof P, Ring J, Nassenstein I, et al. Detection of asymptomatic cerebral microbleeds: a comparative study at 1.5 and 3.0 T. Acad Radiol. 2008;15:895-900.

6. Hamill R. Report of a case of melanosis of the brain, cord and meninges. J Nerv Ment Dis. 1908:594.

7. Khalatbari K. Case 141: superficial siderosis. Radiology. 2009; 250(1):292-7.

8. Ohira M, Takao M. Superficial siderosis. Brain Nerve. 2018 Oct; 70(10):1107-13.

9. Wang J, Gong X. Superficial siderosis of the central nervous system: MR findings with susceptibility-weighted imaging. Clin Imaging. 2011 May-Jun;35(3):217-21.

10. Linn J, Herms J, Dichgans M, Bruckmann H, Fesl G, Freilinger T, et al. Subarachnoid hemosiderosis and superficial cortical hemosiderosis in cerebral amyloid angiopathy. Am J Neuroradiol. 2008;29:184-6.
11. Linn J, Halpin A, Demaerel P, Ruhland J, Giese AD, Dichgans M, et al. Prevalence of superficial siderosis in patients with cerebral amyloid angiopathy. Neurology. 2010;74:1346-50.

12. Viswanathan A, Greenberg SM. Cerebral amyloid angiopathy in the elderly. Ann Neurol. 2011;70:871-80.

13. Charidimou A, Peeters A, Fox Z, Gregoire SM, Vandermeeren Y, Laloux $P$, Jäger HR, Baron JC, Werring DJ. Spectrum of transient focal neurological episodes in cerebral amyloid angiopathy: multicentre magnetic resonance imaging cohort study and meta-analysis. Stroke. 2012 Sep;43(9):2324-30.

14. Charidimou $A$, Peeters AP, Jäger R, Fox Z, Vandermeeren $Y$, Laloux $P$, Baron JC, Werring DJ. Cortical superficial siderosis and intracerebral hemorrhage risk in cerebral amyloid angiopathy. Neurology. 2013 Nov 5;81(19):1666-73.

15. Derle E. Iron chelation in treatment of superficial siderosis. Iran J Neurol. 2018 Oct 7;17(4):195-96.

16. Cossu G, Abbruzzese G, Forni GL, Matta G, Pinto V, Ruffinengo U, Oppo V, Marchese R. Efficacy and safety of deferiprone for the treatment of superficial siderosis: results from a long-term observational study. Neurol Sci. 2019 Jul;40(7):1357-61.

17. 17. Vladila AM, Pavel G, Mitrea D, Nica S, Mihailescu G, Davidescu I, Buraga I. Multiple Cerebral Cavernous Malformations - A Case Report. Ro J Neurol. 2013;12(4):209-13.

18. Levy M, Turtzo C, Llinas RH. Superficial siderosis: a case report and review of the literature. Nat Clin Pract Neurol. 2007;3:54-8.

19. Li KW, Haroun RI, Clatterbuck RE, Murphy K, Rigamonti D. Superficial siderosis associated with multiple cavernous malforma-tions: report of three cases. Neurosurgery. 2001;48:1147-50.

20. Ogura T, Kambe A, Sakamoto M, Shinohara Y, Ogawa T, Kurosaki M. Superficial Siderosis Associated with Pineal Cavernous Malformation. World Neurosurg. 2018 Jan;109:230-232. 